\title{
Evaluación clínico radiológica del uso de un nuevo dispositivo para la reducción cerrada de fracturas distales de radio
}

\author{
Clinical radiological evaluation of the use of a new device for \\ the closed reduction of distal radio fractures
}

\begin{abstract}
Rubén Fernando Sáyago, * Guadalupe S García de la Torreł
*Cirujano ortopedista. Traumatólogo Ortopedista adscrito al Servicio de Traumatología y Ortopedia del Hospital General SSA de Navojoa, Sonora; ₹ Jefa del departamento de Salud Pública. Responsable del Campo Disciplinario de Epidemiología. Programa de Maestría y Doctorado en Ciencias Médicas y Odontológicas y de la Salud, UNAM, Facultad de Medicina, Departamento de Salud Pública.
\end{abstract}

\begin{abstract}
Resumen
Las fracturas distales del radio son frecuentes y representan $55 \%$ del total en menores y adultos así como $1.5 \%$ del total de las visitas a urgencias. En los niños, 90\% son manejadas con reducción cerrada y aplicación de yeso; en mayores el tratamiento varía según su tipo, experiencia del médico y estado general del paciente. En este medio es frecuente que la atención primaria se retrase por empírico (persona tratante que sin ser médico atiende pacientes basándose solamente en su experiencia), ocasionando inflamación y edema importante, lo que dificulta la reducción cerrada de la fractura originando la necesidad de reducción abierta. El presente trabajo muestra un dispositivo de acero, diseñado para la reducción cerrada de fracturas distales del radio que se utilizó en hospitales públicos de Navojoa, Sonora, México. Se documentaron treinta pacientes con fractura desplazada distal del radio con una evolución entre uno y siete días, de 2017 a 2019. Se realizaron mediciones radiológicas estándar pre- y postreducción, obteniendo resultados de $96.66 \%$ de los casos con alineación satisfactoria y diferencia estadísticamente significativa entre índices pre- y post- $(p<0.05)$. Un caso requirió tratamiento quirúrgico por mala corrección, no se documentaron complicaciones neurovasculares distales causadas por el mecanismo de reducción. Se realizaron pruebas biomecánicas al mecanismo que mostraron ventajas sobre la técnica tradicional de reducción con los pulgares. Se concluye que este componente para la reducción cerrada de fracturas distales del radio es una herramienta eficaz y que es posible favorecer su utilización.
\end{abstract}

Palabras clave: Dispositivo, fractura distal de radio, edema, empírico, reducción cerrada, disparo.

\begin{abstract}
Distal radius fractures are frequent and represent $55 \%$ of the total in children and adults as well as $1.5 \%$ of the total emergency room visits. In children, $90 \%$ are managed with closed reduction and application of plaster; in adults, treatment varies according to type, experience of the doctor and general condition of the patient. In this setting, it is common for primary care to be delayed due to empirical events, causing significant inflammation and edema, which makes the closed reduction of the fracture difficult, causing the need for open reduction. The present work shows a steel device, designed for the closed reduction of distal radius fractures that was used in public hospitals in Navojoa, Sonora, Mexico. Thirty patients with displaced distal radius fracture with an evolution between one and seven days, from 2017 to 2019, were documented. Standard radiological measurements were performed before and after reduction, obtaining results of $96.66 \%$ of the cases with satisfactory alignment, and statistically significant difference between pre and post indices $(p<0.05)$. One case required surgical treatment for poor correction, no distal neurovascular complications caused by the reduction mechanism were documented. Biomechanical tests were performed on the device that showed advantages over the traditional thumb reduction technique. It is concluded that this device for the closed reduction of distal radius fractures is an effective tool and that it is possible to favor its use.
\end{abstract}

Keywords: Device, distal radius fracture, edema, empirical, closed reduction, shooting.

Correspondencia:

Dr. Rubén Fernando Sayago

Calle Ocampo 203-A, Col: Reforma, 85830, Navojoa, Sonora, México,

E-mail: sayagortopedia@hotmail.com

Recibido: 26-01-2021. Aceptado: 04-05-2021.
Citar como: Sáyago RF, García TGS. Evaluación clínico radiológica del uso de un nuevo dispositivo para la reducción cerrada de fracturas distales de radio. Orthotips. 2021; 17 (2): 98-107. https://dx.doi. org/10.35366/99904 


\section{Introducción}

En los humanos son muy frecuentes las fracturas de los huesos del antebrazo (ulna y radio), en niños alcanzan del 40 al $55 \%$ del total de las fracturas, ${ }^{1,2}$ y entre 60 y $65 \%$ del total de las fracturas del miembro torácico. Entre 66 y $75 \%$ son del tercio distal del antebrazo, y $20 \%$ del tercio medio entre las edades de cuatro a 12 años. ${ }^{3}$ En los adultos con más de 45 años, las fracturas de muñeca representan $25 \%$ de todas las fracturas, siendo entre 1.5 y $2 \%$ de todas las consultas de la sala de urgencias. ${ }^{1,2,4}$

El tratamiento de las fracturas distales de radio en menores generalmente es conservador (90\%) con reducción cerrada e inmovilización con aparato de yeso. ${ }^{5}$ En los mayores, las fracturas distales de radio son completamente diferentes a los niños, no hay remodelación, la calidad ósea, la complejidad de la fractura y las condiciones generales del paciente hacen la diferencia, por lo que el manejo es controversial. ${ }^{5-10}$

Sin perder de vista que el objetivo del tratamiento, que es la reducción anatómica y la función, ésta se puede lograr mediante reducción cerrada, o reducción abierta, ${ }^{9-13}$ la elección del tipo de reducción dependerá de la complejidad de la fractura, la calidad ósea, la experiencia del cirujano, y las condiciones generales del paciente. . $, 9,12,14-17$ En México y Latinoamérica existe la costumbre de acudir en primera instancia con empírico ${ }^{18-20}$ ante cualquier lesión sufrida en alguna extremidad, siendo más frecuente en el medio rural, lo cual retrasa la atención pronta por el ortopedista, y cuando la lesión es una fractura, el empírico lejos de resolverla, la complica, por lo que el paciente acude con el medico después de 24 horas y en ocasiones hasta semanas después de sufrida la lesión, al no obtener alivio a su dolor. Es frecuente que acudan ya con alguna complicación, desde edema de diversos niveles, celulitis, absceso, hasta complicaciones más graves como síndrome compartimental.

Cuando el paciente se presenta a urgencias en estas circunstancias se da prioridad a las complicaciones y cuando sólo hay hinchazón se intenta la reducción cerrada de la fractura bajo sedación, con alguna de las técnicas de reducción. ${ }^{21-23}$ Los métodos más frecuentemente usados son tracción y contratracción, y la otra técnica, aumentar la deformidad en el sitio de la fractura, y con uno o ambos pulgares el médico tratante empuja el fragmento distal hasta ponerlo en contacto término-terminal con el fragmento proximal, posteriormente se corrige la deformidad con lo cual se logra la reducción, pero el edema dificulta la reducción obligando a realizarla de manera abierta.

Ante esta situación se diseñó un dispositivo para lograr la reducción cerrada y tratar de evitar la cirugía al paciente, el objetivo del presente trabajo es exhibir los resultados de pacientes que se documentaron con reducción cerrada con el uso de este mecanismo.

\section{Presentación del caso}

Se trata de un estudio cuasiexperimental (antes y después) del uso de un mecanismo de innovación, diseñado y construido especialmente para la reducción cerrada de fracturas complicadas distales del radio, aplicado a 30 pacientes presentados como una serie de casos, misma que tiene un nivel tres de evidencia.

\section{Descripción del dispositivo}

El mecanismo es de acero inoxidable y consta de 10 partes (Figuras 1A-B y2). Una varilla principal cuadrada
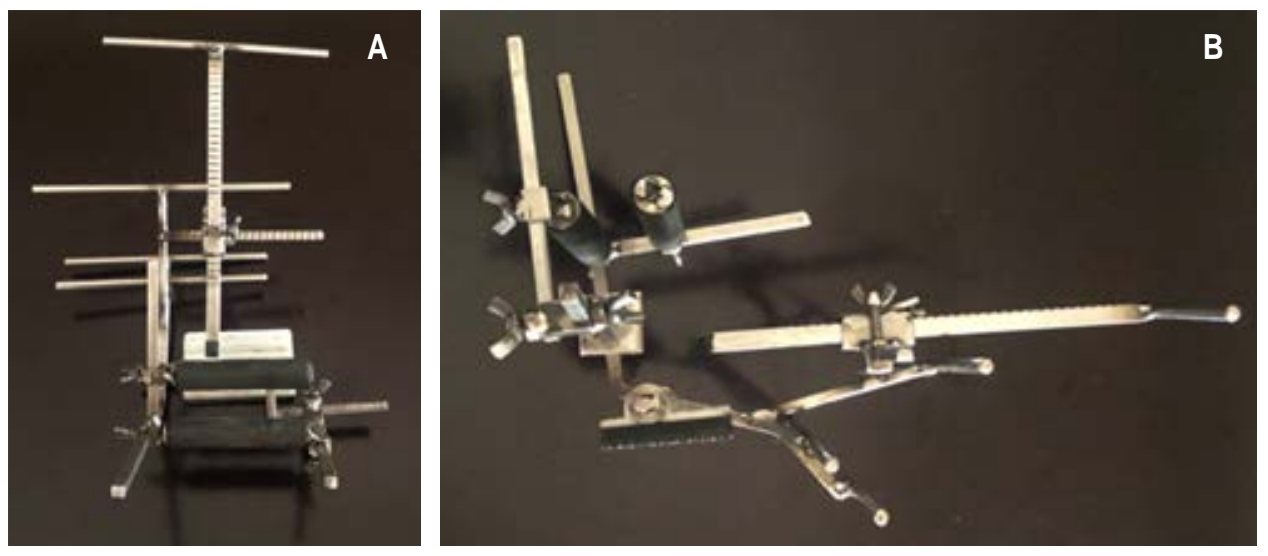

Figura 1:

A) Dispositivo de frente.

B) Dispositivo de lado. 


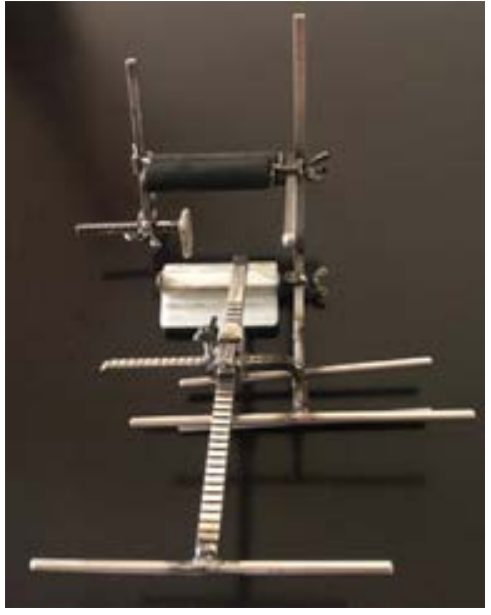

Figura 2:

Mecanismo visto desde arriba

de $1 / 4$ de pulgada de grueso y $30 \mathrm{~cm}$ de longitud, doblada por la mitad a 120 grados, y un segundo doblez a ocho centímetros de la primera angulación, de 30 grados y en sentido inverso a la primera angulación. En el vértice de la segunda angulación se une de manera soldada otra varilla cuadrada de $1 / 4$ de grosor y de nueve centímetros de longitud, formando lo que se le llamará «la columna» del dispositivo, que tiene tres extremos ( $A, A^{\prime \prime}$ y $\left.B\right)$, a esta columna se ensamblan por medio de soldadura (algunas), y otras con candados y opresores, nueve varillas más de acero inoxidable, de $1 / 4$ de grueso y más pequeñas en longitud que la varilla principal o columna. Tiene dos rodillos de plástico industrial, cada rodillo se encuentra dentro de una varilla redonda de acero inoxidable rodeados de polietileno, y un tercer rodillo de plástico industrial, también dentro de otra varilla redonda de acero inoxidable, pero este tercer rodillo, contiene una plataforma rectangular de $8 \times 5$ centímetros, y cubierta de polietileno una de sus caras. En el extremo $A$ " de la columna tiene soldada de manera perpendicular una varilla redonda de $1 / 4$ de pulgada, y forma una "T" con la varilla principal. En el extremo $\mathrm{A}$ se unen perpendicularmente a la columna por medio de soldadura, dos varillas redondas de $1 / 4$ de pulgada de grueso y de 15 cm de longitud, formando otra "T". Cuenta también con otra varilla cuadrada, de $3 / 8$ de pulgada, dentada en una de sus caras, y de $24 \mathrm{~cm}$ de longitud que ha de llamarse «flecha», que en uno de sus extremos forma una "T" con otra varilla redonda de $1 / 4$ de pulgada, y en el otro extremo tiene una cubierta de polietileno de un centímetro por un centímetro, esta flecha se ensambla con un dado hueco que solo permite que la flecha se deslice en una dirección gracias a su cara dentada, formando un sistema ratchet (trinquete). Todo el sistema ratchet se ensambla a la varilla principal por medio de candado y opresor, también hay otra varilla que sujeta la mano y/o muñeca del paciente.

El diseño y la configuración espacial de todo el dispositivo ensamblado es para poder colocar en el mecanismo la extremidad fracturada a reducir, cabe mencionar que éste tiene la versatilidad de adaptarse al tamaño, y sea derecha o izquierda la extremidad a tratar; tiene topes de seguridad para evitar rebasar ciertos límites que puedan causar daño al paciente durante la reducción, es un aparato creado y diseñado

Figura 3:

A) Ubicar el sitio de fractura. B) configurar el artefacto y colocar la muñeca en el aparato y fijarlo.
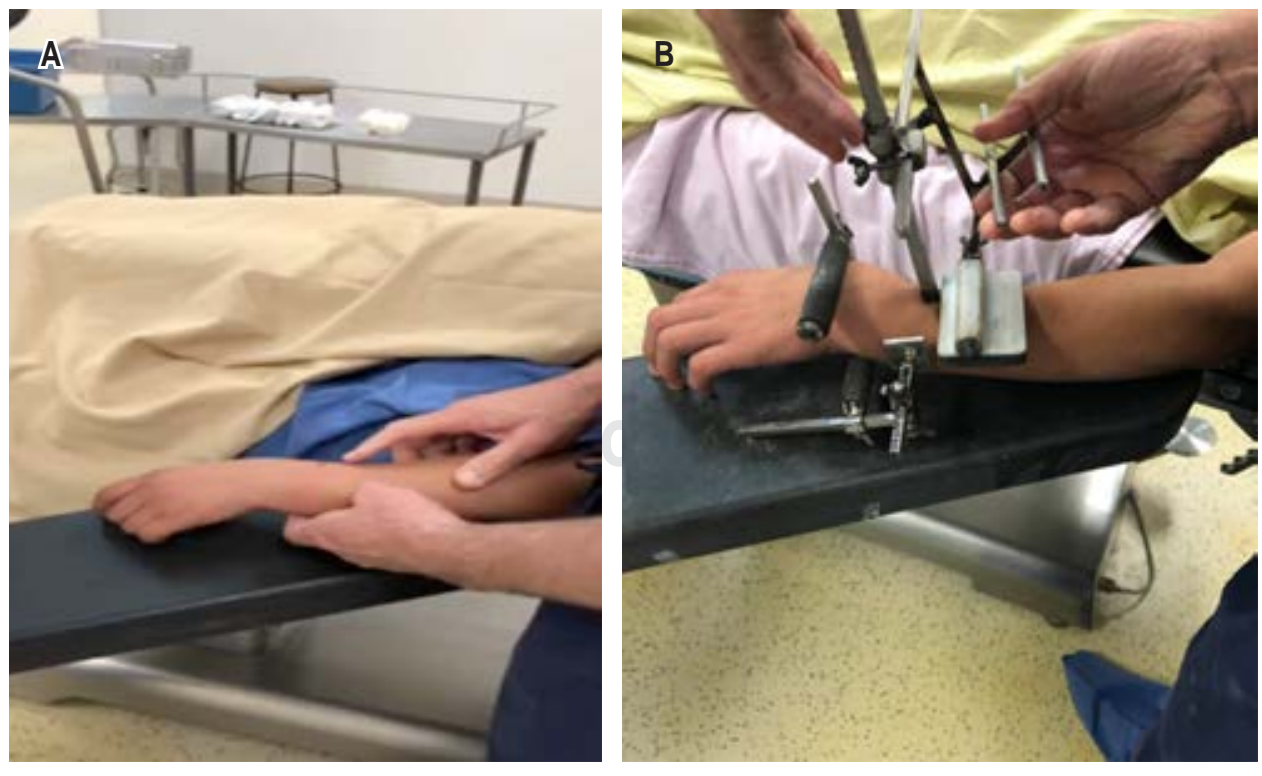


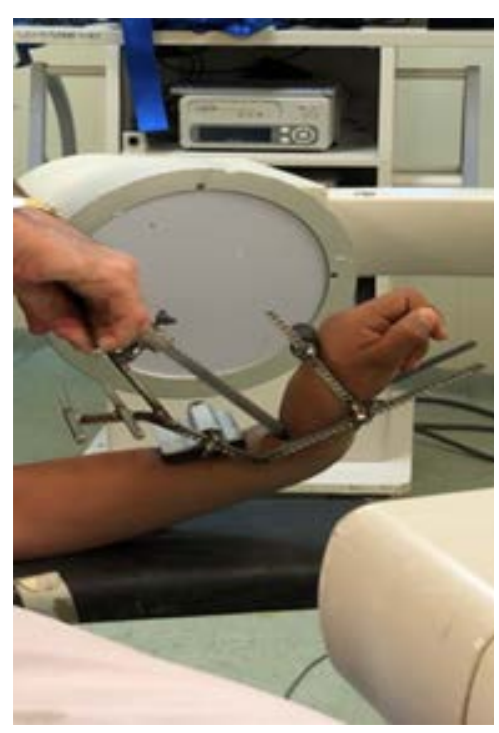

Figura 4:

Angulación de la fractura.

en Navojoa, Sonora, por lo tanto mexicano $100 \%$ y en trámite de patente, no es automatizado, su manejo es manual por el médico tratante y con el paciente bajo anestesia, ya sea bloqueo supraclavicular, o sedación, y se utilizó el arco en $\mathrm{C}$, o mejor conocido como fluoroscopio ${ }^{24}$ cuando fue posible por disponibilidad, lo que evidenció la eficacia mecánica del aparato.

La técnica de reducción consiste en cuatro fases que se describen a continuación:

\section{Primera fase}

Configurar el dispositivo de manera individual al tamaño del miembro afectado, sea derecha o iz- quierda la extremidad, localizar el sitio de la fractura y direccionar la flecha en ese sitio. Una vez configurado el aparato, colocar la extremidad y fijarlo con el sujetador de mano y/o muñeca (Figura 3).

\section{Segunda fase}

Aumentar la deformidad en el sitio de la fractura hasta un límite superior de 90 grados (tiene tope de seguridad por la "T" del extremo A de la columna). Incrementar la deformidad se logra apoyando la plataforma cuadrada de ocho por cinco centímetros en la parte dorsal del antebrazo de la extremidad, y empujando de la parte horizontal de la "T" del extremo A" de la columna (Figura 4).

\section{Tercera fase}

Empujar el fragmento distal de la fractura hasta poner en contacto ambos fragmentos de manera término-terminal. Lo cual se logra al sujetar el médico tratante, con ambas manos, la parte horizontal de la flecha y la parte horizontal de la "T" del extremo A", y al cerrar ambas manos empuja la flecha, cuya dirección y punto exacto donde ésta hará la presión ya fue definido en la primera fase, y por el sistema ratchet que tiene la flecha sólo se desliza en una dirección. El avance de la flecha será paso a paso, siguiendo los dientes de la cara dentada de la fecha. Entre un diente y otro diente hay una distancia de $0.5 \mathrm{~cm}$, por lo tanto, cada avance de la flecha hace un "clic" al que se llamará «disparo» (Figura 5).
Figura 5:

A) Empujar el fragmento distal con ambas manos.

B) Acercamiento de la imagen del arco en $\mathrm{C}$.

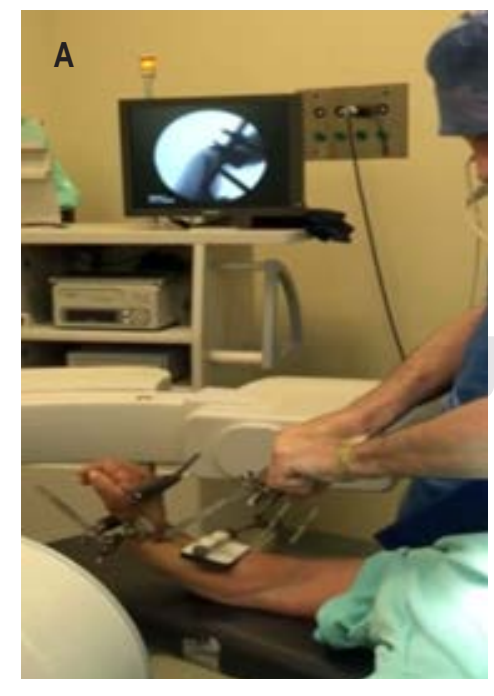

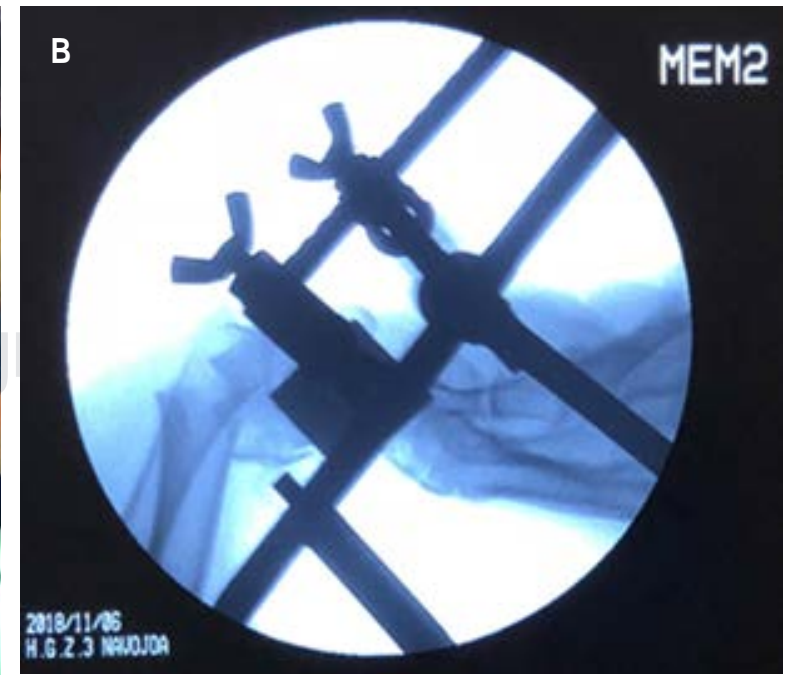




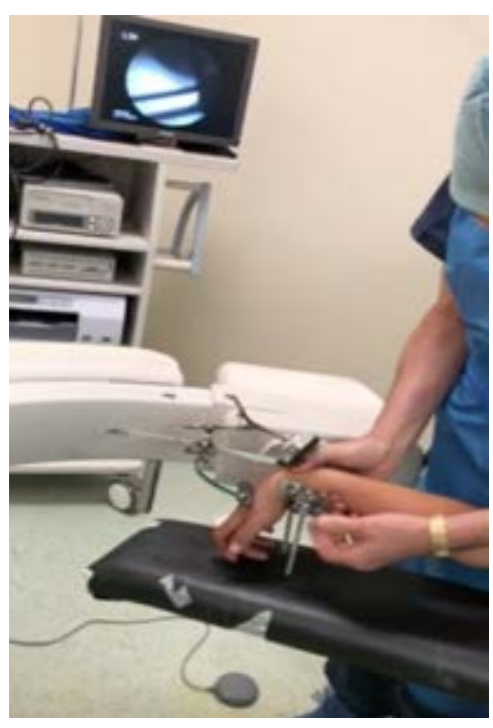

Figura 6:

Eliminar la deformidad de la fase 2, con lo que se logra la reducción y retirar el dispositivo.

\section{Cuarta fase}

Eliminar la deformidad con la que se inició la fase 2 , y con esto se habrá logrado la reducción y se procederá a retirar la extremidad del dispositivo, lo cual se logra oprimiendo el botón que libera la flecha del sistema ratchet y permite que ésta se deslice en dirección contraria. Se retira el sujetador de la mano y/o muñeca, con lo que la extremidad puede retirarse del mecanismo manteniendo la reducción lograda con los dedos del médico tratante (Figura 6).

Los criterios de selección de los pacientes candidatos a reducción cerrada con el presente aparato son:

1. Pacientes adultos con fractura cerrada distal de radio o de Colles ${ }^{4,11}$ extraarticulares tipo A2.2, A3.2, de la clasificación AO, de más de 24 horas de evolución hasta siete días.

2. Pacientes menores con fractura cerrada de metáfisis distal de radio desplazada, ${ }^{25}$ con vértice volar, cabalgada hacia dorsal, de más de 24 ho- ras de desarrollo hasta siete días. Con o sin fractura de la ulna distal.

3. Pacientes niños con epifisiolistesis distales de radio tipo II de Salter y Harris, ${ }^{26}$ cerrada, con evolución de 24 horas o hasta siete días.

4. Pacientes con edema $\geq 2$, positivos del signo de Godet.

Los criterios de exclusión son:

1. Pacientes infantiles 0 adultos con fractura abierta distal de radio.

2. Fracturas intraarticulares.

3. Fracturas con compromiso neurovascular.

4. O que no sea de las características de las fracturas de inclusión.

Con base en los criterios de selección, se atendieron 15 pacientes menores y 15 pacientes mayores, entre 2017 y 2019 , en hospitales públicos y privados en Navojoa, Sonora, México. A cada paciente se le tomaron radiografías pre- y postreducción anteroposterior y lateral, y se realizaron las mediciones radiológicas estándar para adultos: inclinación radial en proyección AP y lateral, altura radial y varianza ulnar. En niños: angulación radial en la proyección AP y la proyección lateral, cabalgamiento, y varianza ulnar. Los datos clínicos incluidos fueron edad, sexo, días de evolución, tratamiento previo, edema de la muñeca (signo de Godet).

El método de fijación de todos los pacientes fue con yeso circular con tres puntos de apoyo por arriba del codo, 3,5,11,15 el manejo inmediato postreducción fue elevación estricta de la extremidad, antiinflamatorios y analgésicos IV, y vigilancia estrecha del paquete neurovascular distal, por 24 a 48 horas dependiendo de la progresión.

Se tomaron radiografías de control las primeras tres semanas para evaluar la reducción, el yeso se dejó por espacio de un mes en los infantes y seis semanas en los adultos, con liberación del codo

Tabla 1: Resultados estadísticos adultos.

\begin{tabular}{lccccccc}
\hline & Mediana pre-reducción & Mínimo & Máximo & Mediana post-reducción & Mínimo & Máximo & $p$ \\
\hline Radiografía anteroposterior & $15^{\circ}$ & $7^{\circ}$ & $21^{\circ}$ & $21^{\circ}$ & $10^{\circ}$ & $26^{\circ}$ & 0.001 \\
RX lateral & $-18^{\circ}$ & $27^{\circ}$ & $-10^{\circ}$ & $10^{\circ}$ & $5^{\circ}$ & $20^{\circ}$ & 0.001 \\
D radial (mm) & 7 & 0 & 15 & 11 & 0 & 15 & 0.022 \\
Varianza ulnar (mm) & 0 & -3 & 7 & -3 & -4 & 2 & 0.001 \\
\hline
\end{tabular}


Sáyago RF et al. Dispositivo para reducir fracturas distales de radio

Tabla 2: Resultados estadísticos niños.

\begin{tabular}{lccccccc}
\hline & Mediana pre-reducción & Mínimo & Máximo & Mediana post-reducción & Mínimo & Máximo & $p$ \\
\hline Radiografía anteroposterior & $0^{\circ}$ & $0^{\circ}$ & $20^{\circ}$ & $0^{\circ}$ & $0^{\circ}$ & $0^{\circ}$ & 0.042 \\
RX lateral & $20^{\circ}$ & $0^{\circ}$ & $38^{\circ}$ & $0^{\circ}$ & $0^{\circ}$ & $4^{0}$ & 0.001 \\
Cabalgada (mm) & 15 & 0 & 20 & 0 & 0 & 0 & 0.005 \\
Varianza ulnar (mm) & -1 & -3 & 7 & -3 & -3 & -1 & 0.003 \\
\hline
\end{tabular}

a las cuatro semanas, posteriormente se retiró el aparato de yeso y se inició la rehabilitación de todos los pacientes, con un seguimiento mínimo de tres meses y máximo de 10. La evaluación radiológica y funcional final se realizó mediante la escala de Gartland y Werley. ${ }^{24}$

Las pruebas biomecánicas a la técnica de reducción, con los pulgares y con el dispositivo, se realizaron con dinamómetro electrónico para mano o empuñadura, marca Camry, modelo EH101. Se sometieron pruebas por diferentes internos y médicos del hospital a ejercer fuerza en el dinamómetro con un pulgar 10 pruebas, con dos pulgares 10 pruebas, y con las dos manos 10 pruebas, y se registraron los datos. ${ }^{23}$

Se explicó a los pacientes y a familiares de éstos la técnica que se usaría para la reducción de sus fracturas y en caso de estar de acuerdo se solicitó su firma de consentimiento informado.

El análisis estadístico: a través de su mediana se compararon los momentos pre- y postreducción, mediante la prueba de Wilcoxon y se consideró un valor de $p \leq 0.05$ como estadísticamente significativo.

\section{Resultados}

\section{Adultos}

El promedio de edad fue de 50.6 años, con un rango entre 30 y 60.13 eran mujeres (86.7\%), los otros dos eran hombres. El lado más afectado fue el izquierdo (66.7\%), mientras que el lado derecho fue $33.3 \%$. Según la $A O, 13$ pacientes se clasificaron como A2.2 (86.7\%) y dos como A3.2 (13.3\%). Dos de los pacientes (13.3\%) se presentaron a atención con siete días de evolución, un paciente $(6.7 \%)$ con seis días de evolución, tres pacientes $(20 \%)$ con cinco días, seis pacientes (40\%) con cuatro días y tres pacientes (20\%) con tres días de evolución. En lo referente a la presencia de edema, tres pacientes (20\%) tuvieron $2 x$, siete pacientes $(46.7 \%) 3 x$ y cinco pacientes (33.3\%) 4x. Once de los pacientes (73.3\%) acudieron primero con tratante empírico, tres pacientes $(20 \%)$ no recibieron ningún tratamiento, y sólo un paciente $(6.7 \%)$ se le colocó previamente una férula en el servicio de urgencias.

La mediana de la inclinación radial en proyección AP prerreducción fue de 15 grados (rango de 7 a 21 grados) y la de postreducción fue de 21 grados (rango 10 a 26) con un valor de $p=0.001$.

La mediana de la inclinación radial en proyección lateral prerreducción fue de -18 grados (27 a -10), mientras que la de postreducción fue de 10 grados (5 a 20), $p=0.001$.

La altura radial mediana pre-reducción fue de siete milímetros ( 0 a 15), mientras que la de postreducción fue de $11 \mathrm{~mm}(0$ a 15), $p=0.022$.

La mediana de varianza ulnar prerreducción fue de cero $\mathrm{mm}(-3 \mathrm{a}$ 7) y la de postreducción fue $-3 \mathrm{~mm}$ (-4 a 2), $\mathrm{p}=0.001$ (Tabla 1).

No se presentaron complicaciones neurovasculares, ni de otro tipo, secundarias a la reducción con el dispositivo; un paciente adulto (6.7\%), tuvo mala reducción por lo que terminó en cirugía para reducción abierta, su fractura fue clasificación A3.2 de la $A O$, se trató de una mujer de 52 años con cinco días de evolución, que fue atendida previamente por un empírico, y presentó edema 3x de Godet en el momento de la reducción cerrada; cinco pacientes requirieron recambio de yeso por disminución del edema $(33.3 \%)$, cuatro en la primera semana $(80 \%)$, uno en la segunda semana (20)\%.

En la escala clínica de Gartland y Werley ${ }^{24}$ un paciente adulto $(6.7 \%)$ presentó rigidez de muñeca y dedos y requirió movilización bajo anestesia, posterior a la cual persistió la rigidez en menor medida y alcanzó cuatro puntos en la evaluación objetiva, y en total nueve clasificado como regular, la paciente en cuestión fue una fémina de 46 años de edad con fractura distal de radio izquierda, clasificación A2.2, con siete días de desarrollo, tratamiento previo con empírico, edema Godet $3^{\text {t, }}$ se le cambió el yeso la primera semana por disminución de la hinchazón. 


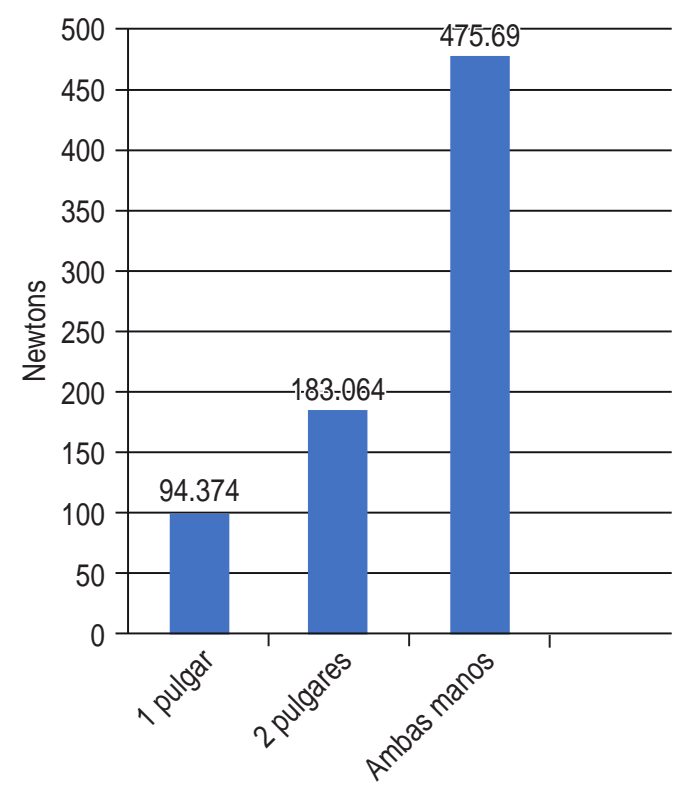

Regular ( 9 a 20 puntos) cinco pacientes (33.3\%), bueno (3 a 8 puntos) nueve pacientes (60\%).

En la escala radiológica de Gartland y Werley:24 excelente 11 pacientes $(73.3 \%)$, bueno tres pacientes (20\%), malo un paciente $(6.7 \%)$.

\section{Niños}

El promedio de edad fue de 10.5 años, con un rango entre 7 y 13 años. En $86.7 \%$ (13) eran varones, el resto, mujeres. En la mayoría de los pacientes (73.3\% que corresponde a 11), el lado afectado fue el izquierdo y $26.7 \%$ el derecho. Se le diagnosticó epifisiolistesis distal de radio a tres pacientes $(20 \%)$, fractura de metáfisis distal de radio a seis $(40 \%)$ y fractura de metáfisis distal de radio y ulna a seis pacientes (40\%). Un paciente $(6.7 \%)$ acudió a consulta después de dos días de evolución, tres pacientes $(20 \%)$ lo hicieron a tres días de la fractura, otros tres $(20 \%)$ después de cuatro días, cinco pacientes (33.3\%) esperaron cinco días y tres (20\%) acudieron después de seis días. En lo referente a la presencia de edema, siete pacientes $(46.7 \%)$ lo tuvieron $2 x$, otros siete (46.7\%) $3 x$, un paciente (6.6\%) 4x. En $86.7 \%$ (13) de los pacientes acudieron previamente con empírico, un paciente más (6.7\%) no recibió ningún tipo de tratamiento previo y en otro paciente $(6.7 \%)$ se realizó reducción cerrada fallida por traumatólogo.

La mediana de la inclinación radial en proyección AP prerreducción fue de cero grados (0 a 20) y la correspondiente a postreducción fue cero grados, sin variación, pues todos los infantes postreducción tuvieron la misma inclinación ( $p=0.042)$.

La mediana de la angulación en proyección lateral pre-reducción fue de 20 grados ( 0 a 38), mientras que la de postreducción fue cero grados ( 0 a 4), $p=0.001$.

La mediana del cabalgamiento prerreducción fue $1.5 \mathrm{~cm}(0 \mathrm{a} 2)$ y postreducción fue cero grados, sin variación, $p=0.005$.

La varianza ulnar mediana en prerreducción fue $-1 \mathrm{~mm}$ (-3 a 7), mientras que ya en postreducción fue $-3 \mathrm{~mm}(-3 \mathrm{a}-1), \mathrm{p}=0.003$ (Tabla 2).

En los niños se logró la reducción radiológica con cero angulación radial en la AP en los 15 pacientes (100\%), con cero angulación en la proyección lateral en 12 pacientes $(80 \%)$, con tres grados en dos pacientes $(13.3 \%)$, cuatro grados en un paciente $(6.7 \%)$, cero cabalgamiento en 15 pacientes (100\%). No se presentaron datos de arresto epifisario en ningún paciente con diagnóstico de epifisiolistesis distal de radio hasta el momento.

Las pruebas biomecánicas que se efectuaron al dispositivo con el dinamómetro fueron:

1. Con un pulgar el promedio fue 94.37 Newton, con un rango de 83.3 a 100.94 Newton.

2. Con dos pulgares el promedio fue $183.06 \mathrm{New}-$ ton con un rango de 172.48 a 190.12 Newton.

3. Con las dos manos el promedio fue 475.69 Newton, con un rango de 408.66 a 594.86 Newton (Figura 7). 


\section{Discusión}

Las fracturas distales de radio en menores y adultos, además de ser las más frecuentes,,$^{1,2}$ tienen diversos niveles de complejidad para su reducción por sí mismas, ${ }^{14}$ y si a estos factores inherentes a la lesión se le agregan otros, como el retraso en la atención médica y la intervención de empíricos, la complejidad para su reducción aumenta considerablemente, ${ }^{18-20}$ ya que la demora en la atención en caso de una fractura, más la manipulación por empírico, son fuentes de hinchazón adicional al edema natural que ocurre en cualquier fractura.

En el presente trabajo se enfatiza la importancia que esta situación tiene en la evolución de las fracturas, por un aspecto cultural de usos y costumbres, y que no hay estadísticas en México en este sentido. Los resultados muestran que $73.3 \%$ de los mayores y $86.7 \%$ de los menores fueron atendidos previamente por un empírico, es entendible que sea mayor el número en niños ya que son los padres quienes lo deciden, la relación directa de esta costumbre con la hinchazón resultante es también muy clara, siendo el signo de Godet 3x de $46.7 \%$ en infantes y adultos, siendo los adultos los que presentaron mayor edema (Godet 4x) que representó 33.33\%, comparado con el $6.7 \%$ en los niños, así como los días de desarrollo en el que acudieron a urgencias, que fue de cuatro días en los adultos $40 \%$, y en los menores fue de cinco días 33.3\%, por lo demás, la distribución por sexo, edad, y el lado afectado es muy similar a los datos reportados en la literatura mundial. ${ }^{1,2,14}$

Como se mencionó previamente, el presente trabajo sólo está enfocado en la técnica de reducción cerrada, no intenta analizar el tipo de método de fijación de la reducción, lo cual queda a juicio y experiencia del médico tratante, ya sea con molde de yeso, que fue la técnica que se utilizó en este trabajo, pero puede ser con clavillos cruzados o fijadores externos, y puede ser que la técnica utilizada para estos pacientes sea la que influyó en que no hubiera ningún paciente con resultado excelente en la evaluación clínica según la clasificación de Gartland y Werley, ${ }^{24}$ sin embargo, en la evaluación radiológica de la misma escala sí hubo
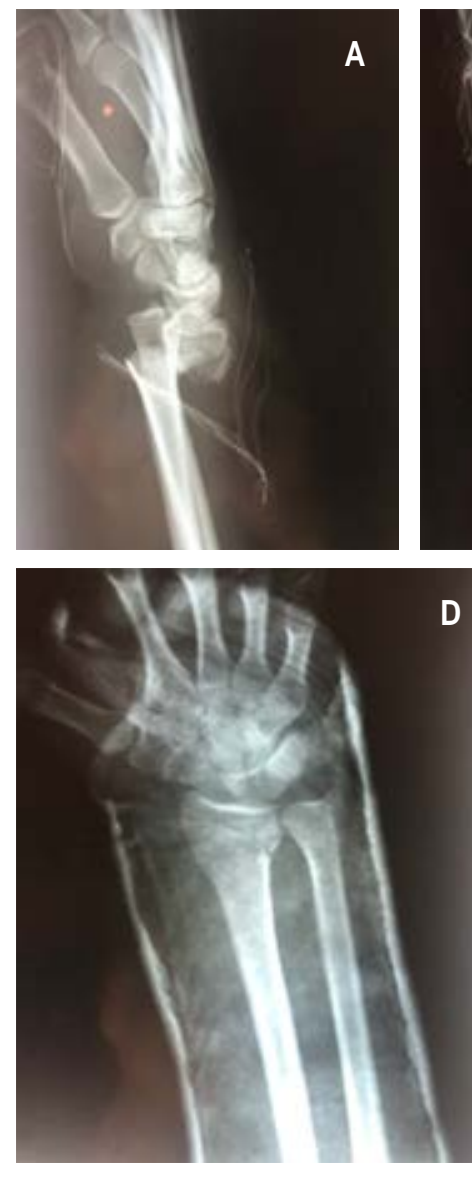

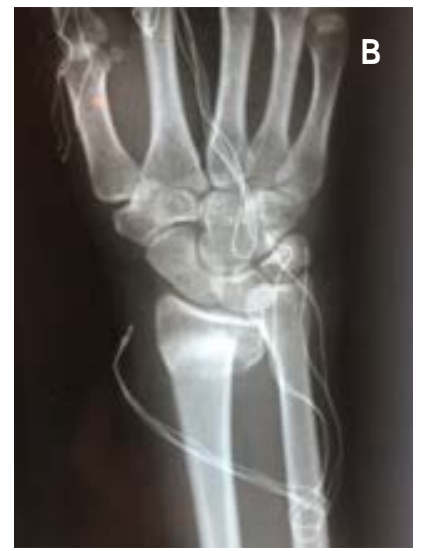

D

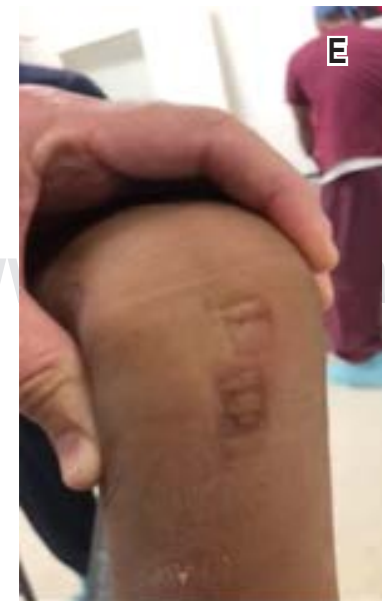

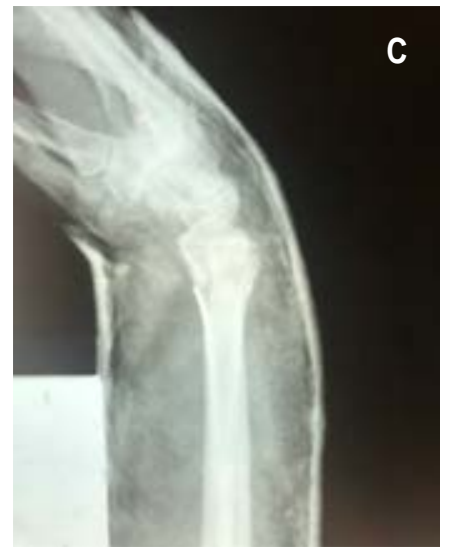

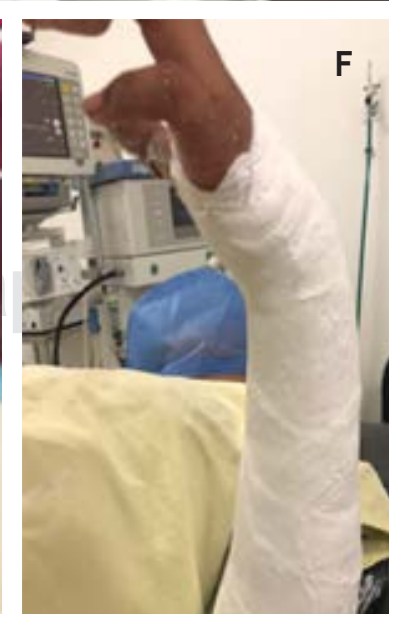

Figura 8:

Femenino de 40 años de edad, tres días de evolución, tratamiento previo con empírico Godet 4x. A) Rx lateral prerreducción. B) Rx Ap prerreducción. C) $\mathrm{Rx}$ lateral postreducción. D) Radiografía anteroposterior postreducción. E) La marca del aparato en la piel por la fuerza aplicada. F) Reducción ya con el molde de yeso. 

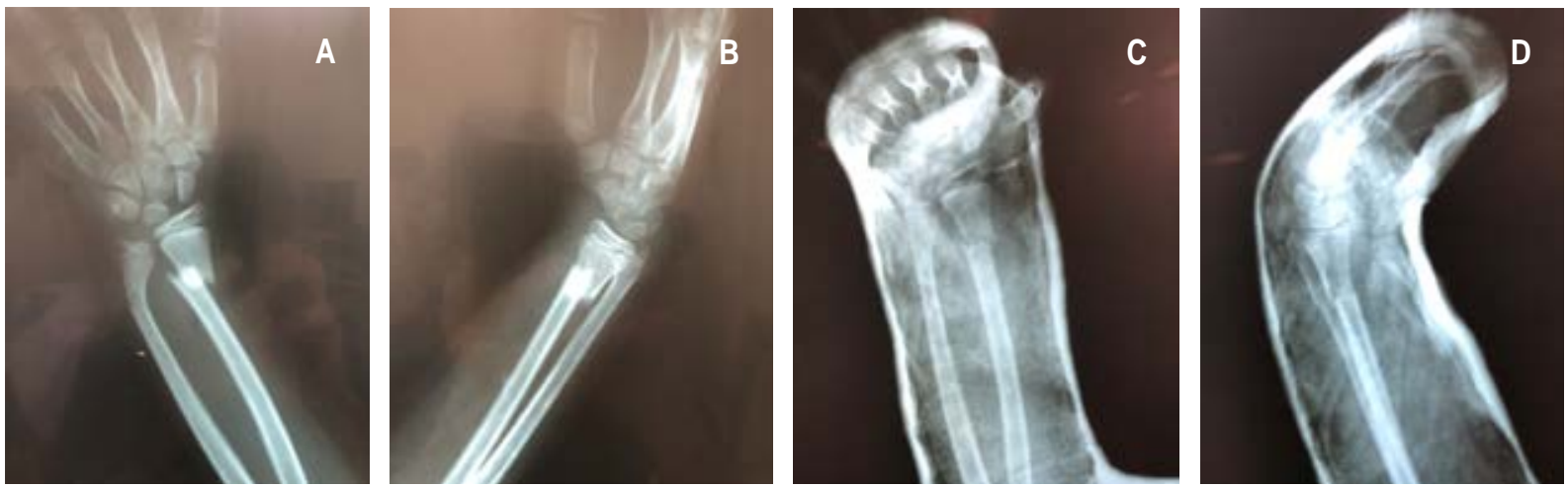

Figura 9: Femenino de ocho años de edad con cuatro días de desarrollo, tratamiento previo con empírico, Godet 3x. A) Radiografía anteroposterior prerreducción. B) Radiografía lateral prerreducción. C) Radiografía anteroposterior postreducción. D) Radiografía lateral postreducción.

resultados excelentes en $73.3 \%$, y fue evidente que los niños fueron los que tuvieron mejores resultados, debido probablemente a la mayor complejidad de las fracturas en los adultos.

Los resultados estadísticos muestran que la probabilidad de que los cambios obtenidos en las mediciones radiográficas pre- y post- sean debidos al azar es lo suficientemente baja, por lo que se puede afirmar que fue el dispositivo el que logró esos cambios (Figuras 8 y 9).

En las pruebas biomecánicas realizadas con dinamómetro se ubica el mecanismo muy superior a la técnica de uno o dos pulgares, ya que el aparato logra la misma reducción que utilizando ambas manos, y en las diversas técnicas existentes como son tracción con los dedales, tracción contratracción, y LEAFR $^{21-23}$ también se coloca superior a la tracción con los dedales que muestran una fuerza de 443.8 Newton, otra ventaja del artefacto es que logra la reducción sin necesidad de ayudante, sólo manejado por el médico tratante.

\section{Conclusiones}

Se lograron buenos resultados con el dispositivo, a pesar de que la muestra es pequeña (30 pacientes), el aparato mostró ser una herramienta útil en ese tipo de fracturas y se puede promover su uso con la intención de evitarle una cirugía al paciente con todos los riesgos inherentes, y bajar los costos hospitalarios, el siguiente estudio con el mecanismo sería un estudio prospectivo, comparativo con la técnica tradicional (ensayo clínico controlado), lo que seguramente arrojaría información importante.

\section{Referencias}

1. Nellans KW, Kowalsaky E, Chung KC. The epidemiology of distal radius fractures. Hand Clin. 2012; 28 (2): 113-125.

2. Wood AM, Robertson GA, Renie L, et al. The epidemiology of sport-related fractures in adolescent. Injury. 2010; 41 (8): 834-838.

3. Pountos I, Clegg J, Siddiqui A. Diagnosis and treatment of greenstik and tours fractures of the distal radius in children: A prospective randomized single blind study. J Child Orthop. 2010; 4 (4): 321-326.

4. Colles A. On the fracture of the carpal extremity of the radius. Edinb Med Surg J, 1814; 10: 181.

5. Chung KC, Shauver MJ, Birkmeyer JD. Trends in the United Estates in the treatment of distal radial fractures in the ederly. J Bone Joint Surg Am. 2009; 91 (8): 1868-1873.

6. Pedersen J, Oksbjerre MS, Duedal RJ, et al. A protocol for a single-center, single-blinded randomized-controlled trial investigating volar plating versus conservative treatment of unestable distal radius fractures in patients older than 65 years. BMC Muskuloskelet Disord. 2019; 20 (1): 309.

7. Martinez-Mendez D, Lizaur-Utrilla A, de-Juan-Herrera J. Intra-articular distal radius fracturesin elderly: a randomized prospective study casting versus volar plating. J Hand Surgery. 2018; 43 (2): 142-147.

8. Aparicio P, Izquierdo O, Castellanos J. Conservative treatment of distal radius fractures: a prospoctive descriptive study. Hand (NY). 2018; 13 (4): 448-454.

9. Rangel RR, Duran MN, Matuz JJ. Evaluacion clínico-radiológico de fracturas distales de radio tratadas con técnica percutánea. Acta Ortop Mex. 2010; 24 (3): 169-176.

10. Walenkamp MM, Carel GJ, Beumer A, et al. Surgery versus conservative treatment in patients with type $A$ distal radius fractures, a randomized controlled trial. BMC Musculoskelet Disord. 2014; 15 (1): 90.

11. Serrano CM. Fracturas distales de radio clasificación y tratamiento conservador. Revista Española Cirugía Osteoarticular. 2008; 46 (236): 141-154.

12. Chung KC, Shauver MJ, Yin H, et al. Variations in the use of internal fixation for distal radial fracture in the United States medicare population. J Bone Joint Surg Am. 2011; 93 (23): 2154-2162. 
13. Seely MA, Fabricant PD, Lawrence JT. Teaching the basics: development and validation of a distal radius reduction an casting model. Clin Orthop Relat Res. 2017; 475 (9): 2298-2305.

14. Clayton RA, Gaston MS, Ralston SH, et al. Association between decreased bone mineral density and severity of distal radial fractures. J Bone Joint Surg Am. 2009; 91 (3): 613-619.

15. Yao Sen Wu, Jie Yang, Lin Zhen, et al. Factors associated with the decision for operative versus conservative treatment of displaced distal radius fractures in the elderly. Anz J Surg. 2019; (89): E428-E432.

16. Waljee JF, Zhong L, Shauver MJ, et al The influence of surgeron age on distal radius fracture treatment in the United States a population-based study. J Hand Surg Am. 2014; 39 (5): 844-851.

17. Dialey SK, Miller AR, Kakasu R, Wyrick JD, Stern PJ. The effectiveness of mini-C-arm fluoroscopy for the closed reduction of distal radius fractures in adults: A randomized controlled trial. J Hand Surg. 2018; 43 (10): 927-931.

18. Marie-Jeanne DC. Protección y Cura Medicina Tradicional en comunidades negras de la costa chica Oax. México D.F. Programa Universitario de Estudios de la Diversidad cultural y la interculturalidad. 2017; 1 (9): 35-53.

19. Anderson R. The treatment of musculoskeletal disorders by a Mexican bonesetter (SOBADOR). Soc Sci Med. 1987; 24 (1): 43-46.
20. Huber BR, Anderson R. Bonesetters and curers in a Mexican community: Conceptual models, status, and gender. Med Anthropol. 1996; 17 (1): 23-38.

21. Liles R, Fierson JN, Wolf $\mathrm{Cl}$, et al. Reduction of Colles' fracture by weight traction under local anesthesia. South Med J. 1969; 62 (1): 45-48.

22. Earnshaw SA, Aladin A, Surendran S, et al. Closed reduction of Colles fractures: comparison of manual manipulation and finger-trap traction: a prospective, randomized study. J Bone Joint Surg Am. 2002; 84 A: 354-358.

23. Eichinger JK, Aguchukwu U, Franklin J, et al. A new reduction technique for completly displaced forearm and wrist fractures in children: a boimechanical assesment and 4-year clinical evaluation. J Pediatr Orthp. 2001; 31 (7): e73-e79.

24. Gartland JJ Jr, Werley CW. Evaluation of healed Colles' fractures. J Bone Surg Am. 1951; 33-A(4): 895-907.

25. Solongo T, Audige L, Clavert JM, et al. The AO comprehensive classification of pediatric long-bone fractures: a web-based multicenter agreement study. J Pediatr Orthop. 2007;27: 171-180.

26. Salter K, Harris WR. Injuries involving the epiphyseal plate. J Bone Joint Surg Am. 1963; 45 (45): 587-622.

\section{Conflicto de intereses}

\section{Este artículo no tiene conflicto de intereses.}

